\title{
Editorial
}

\section{Nanoscale Biological Materials}

\author{
Kilho Eom, ${ }^{1}$ Serdal Kirmizialtin, ${ }^{2}$ Yaling Liu, ${ }^{3}$ and Zhiping $\mathrm{Xu}^{4}$ \\ ${ }^{1}$ Biomechanics Laboratory, College of Sport Science, Sungkyunkwan University (SKKU), \\ Suwon 16419, Republic of Korea \\ ${ }^{2}$ Chemistry Program, New York University Abu Dhabi, Abu Dhabi, UAE \\ ${ }^{3}$ Department of Mechanical Engineering and Mechanics, Lehigh University, \\ Bethlehem, PA 18015, USA \\ ${ }^{4}$ Department of Engineering Mechanics, Tsinghua University, Beijing 100084, China
}

Correspondence should be addressed to Kilho Eom; kilhoeom@skku.edu

Received 27 July 2016; Accepted 27 July 2016

Copyright (C) 2016 Kilho Eom et al. This is an open access article distributed under the Creative Commons Attribution License, which permits unrestricted use, distribution, and reproduction in any medium, provided the original work is properly cited.

Biological materials at nanoscale such as proteins, antibodies, lipids, and nucleic acids have been highlighted not only for their important roles in life sciences but also for their wide range of applications in engineering and materials science. Understanding the design principles of biomaterials allows for gaining great insight into the creation and control of novel materials with interesting functional properties. Therefore it is of great importance to characterize the biomaterials' microstructures, self-assembly mechanism, and dynamics in high spatial and temporal resolution. During the last two decades, with technological advances in experimental instrumentation and methodology (e.g., single-molecule experiments) and advances in hardware and software that lead to more realistic computer simulations (e.g., atomistic, coarse-grained, and continuum simulations), the structures, properties, functions, and dynamics of nanoscale biological materials have been extensively studied. For instance, single-molecule experiments and atomistic simulations have revealed the underlying mechanisms of protein folding, protein dynamics, protein aggregation, RNA/DNA unzipping, and interactions between nanoscale biological materials (e.g., protein) with other chemical compounds (e.g., drug molecule). Understanding of these fundamental processes at atomic scale has driven multiple applications in biomedical engineering such as developing functional biomaterials with rational design.

With the advancements in the area of nanoscale biological materials as described above, this special issue is aimed towards presenting the current state-of-the-art approaches in understanding the structures and dynamics of biological materials as well as their relationship with materials properties and hence the functions of the nanoscale biological materials including, but not limited to, DNA, RNA, protein, lipid, and self-assembled structures formed from these building blocks. This special issue covers different aspect of characterization of nanoscale biological materials from both computational and experimental viewpoints.

As a first example of these nanoscale biological materials, amyloid fibrils are discussed in this issue. Amyloid fibrils are a hallmark of neurodegenerative diseases, yet they have received great attention due to their remarkable structural stability. The mechanical strength of fibrils is comparable to that of mechanically strong protein materials such as spider silk. In the review paper entitled "Nanomechanical Characterization of Amyloid Fibrils Using Single-Molecule Experiments and Computational Simulations," B. Choi et al. described the recent efforts to characterize the mechanical properties of amyloid fibrils using single-molecule experiments (e.g., atomic force microscopy-based experiments) and computer simulations (e.g., atomistic and coarse-grained simulations). As described in the review paper, experimental and computer simulation approaches provide an estimate of elastic modulus for amyloid fibrils to be in the order of 1 to $10 \mathrm{GPa}$, which is comparable to the elastic modulus of a spider silk protein that is renowned as a mechanically strong protein material. M. Lee et al., in their paper "Effects of End-Terminal Capping on Transthyretin (105-115) Amyloid Protofibrils Using Steered Molecular Dynamics," 
explored the stability and mechanical properties of amyloid fibrils made of transthyretin (TTR). Using steered molecular dynamics simulations they provide great insight into the underlying mechanisms of the end-terminal capping effect on the fibril stability and the mechanical response of TTR amyloid fibrils. While deoxyribonucleic acid (DNA) is an important biomolecule that performs physiological functions such as delivering the genetic information, it has recently been considered as a building block for developing novel large-scale functional structures such as DNA origami. The mechanical properties (e.g., flexibility) of individual DNA molecules are known to play key role in the process of forming DNA origami structures. In a paper entitled "Sensitivity Analysis for the Mechanical Properties of DNA Bundles," Y.J. Kim and D.-N. Kim studied the role of the mechanical properties of DNA bundles in forming the three-dimensional structural motifs such as DNA junctions and strand breaks by performing sensitivity analysis on a six-helix DNA bundle structure using finite element modeling approaches.

Functionalization of nanomaterials with biological molecules has played a vital role in developing a nanobiointerface platform, which can be used in many ways such as for biosensing or drug delivery. J. Nam et al., in their paper entitled "Lipid Reconstitution-Enabled Formation of Gold Nanoparticle Clusters for Mimetic Cellular Membrane," delineated the formation of gold nanoparticle clusters encapsulated with reconstituted phospholipid bilayers for facilitating cellular uptake, a process necessary for nanomaterialbased drug delivery. J. Jung, in her review paper entitled "Emerging Utilization of Chrysin Using Nanoscale Modification," discussed the current state-of-the-art approaches in nanoencapsulation and conjugation for overcoming the utilization of chrysin in pharmaceutical applications. In a paper, entitled "Optimal Synthesis of Horizontally Aligned Single-Walled Carbon Nanotubes and Their Biofunctionalization for Biosensing Applications," D. Jung et al. discussed how carbon nanotubes can be used to capture diseaserelated biomolecules in high sensitivity. They presented the detailed optimal synthesis conditions for such horizontally aligned carbon nanotube arrays functionalized with chemical molecules for biosensing and optical applications. Moreover, nanoscale friction and adhesion are one of the most important physical parameters in designing a nanobiointerface system and a challenging phenomenon to explain using physical principles. S. Y. Baek and K. Kim, in their paper entitled "Development of a Time-Dependent Friction Model for Frictional Aging at the Nanoscale," presented the theoretical model of nanoscale frictional aging based on a cohesive zone model. Their model was validated using numerical simulations and was used successfully to interpret the recent experimental data.

The guest editors hope that this special issue can inspire the readers and stimulate further research in the area of nanoscale biological materials, in particular, the characterization of the relationship between their structures, properties, and functions. These studies will doubtlessly lead to the exploration of novel applications in engineering and materials science while they further elucidate the physical principles behind the properties of biomaterials at nanoscale.

\section{Acknowledgments}

The editors are grateful to the authors for their contributions to this special issue and the anonymous reviewers for their fruitful discussions and comments.

Kilho Eom Serdal Kirmizialtin Yaling Liu Zhiping Xu 

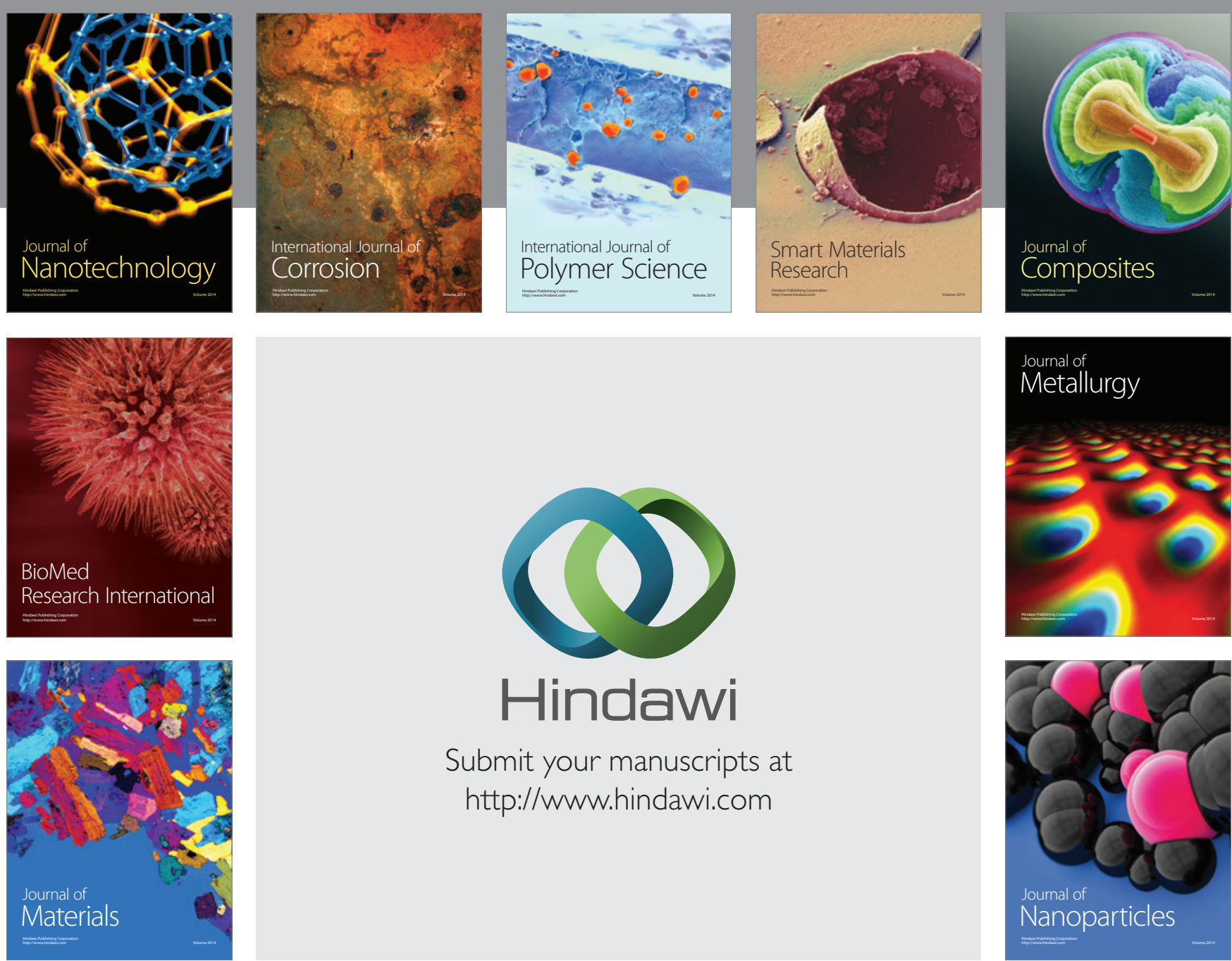

\section{Hindawi}

Submit your manuscripts at

http://www.hindawi.com

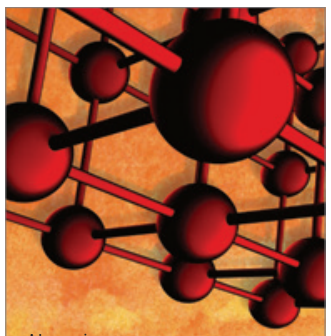

Materials Science and Engineering
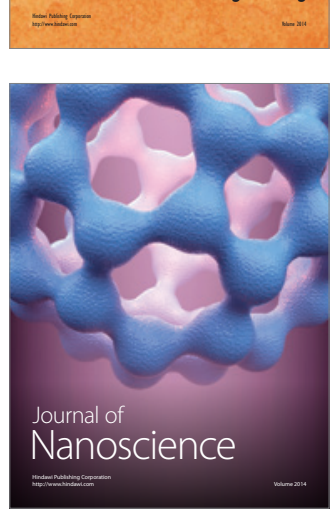
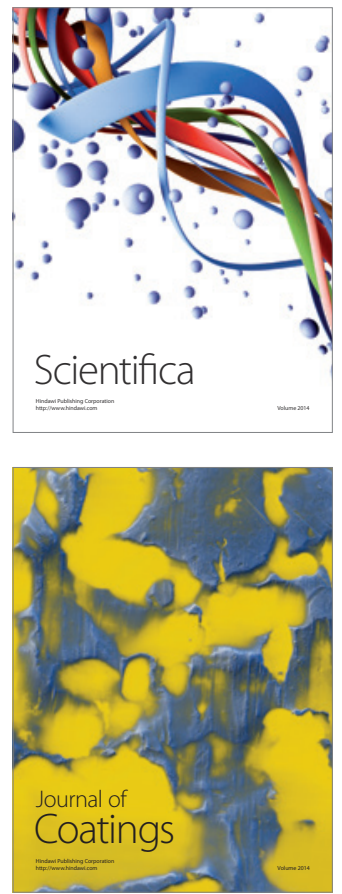
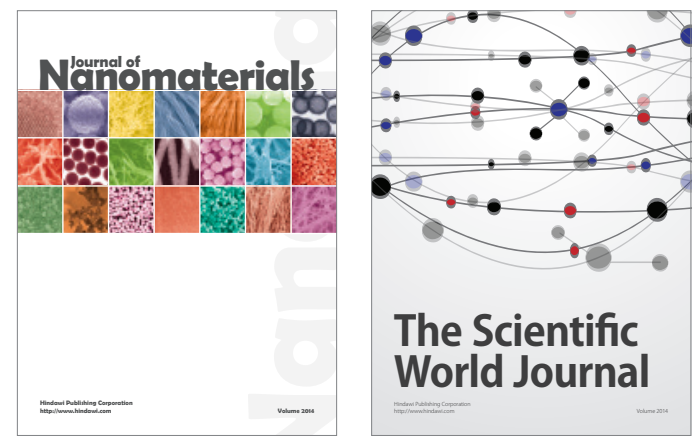

The Scientific World Journal
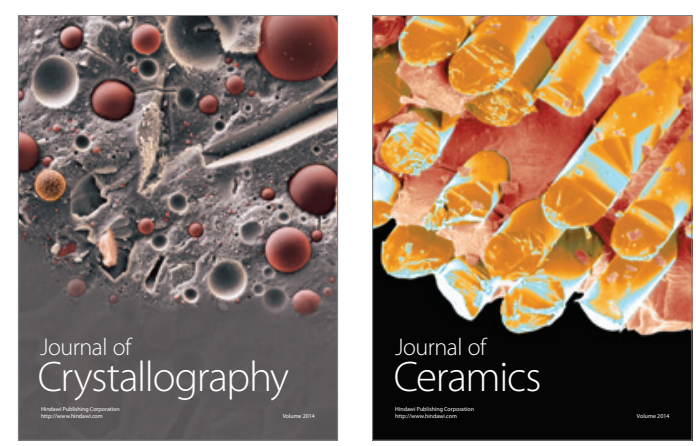
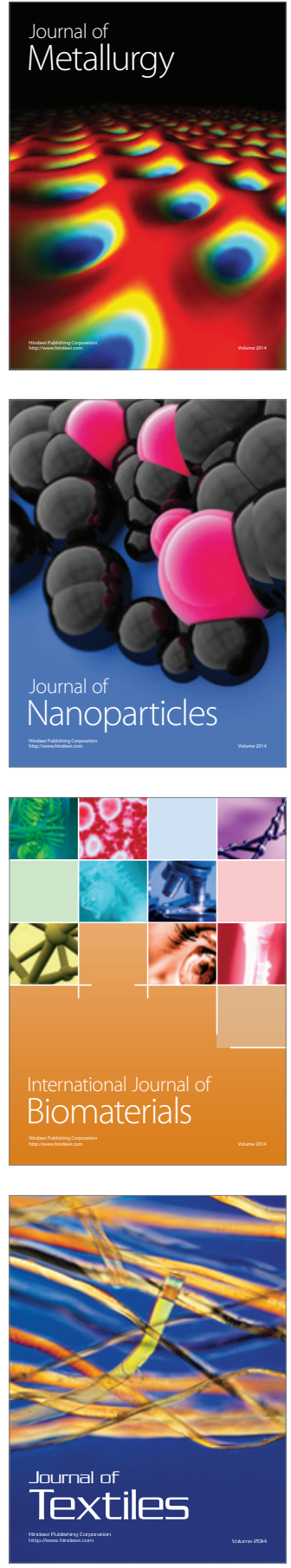